\title{
On the Bisection Method for Triangles
}

\author{
By Andrew Adler
}

\begin{abstract}
Let $U V W$ be a triangle with vertices $U, V$, and $W$. It is "bisected" as follows: choose a longest edge (say $V W$ ) of $U V W$, and let $A$ be the midpoint of $V W$. The $U V W$ gives birth to two daughter triangles $U V A$ and $U W A$. Continue this bisection process forever.

We prove that the infinite family of triangles so obtained falls into finitely many similarity classes, and we obtain sharp estimates for the longest $j$ th generation edge.
\end{abstract}

1. Introduction. Let $U V W$ be the triangle with vertices $U, V$, and $W$. We "bisect" triangles as follows: choose a longest edge (say $V W$ ) of $U V W$, and let $A$ be the midpoint of $V W$. Then $U V W$ gives birth to two daughter triangles $U V A$ and $U A W$. So the generation 0 triangle $U V W$ gives rise to two generation 1 triangles. "Bisect" these in turn, giving rise to four generation 2 triangles, and so on. So $U V W$ through this process gives rise to an infinite family of triangles. This bisection process and a generalization to three dimensions have a number of numerical applications; see, e.g., [1], [3], [4].

Let $m_{j}$ be the length of the longest $j$ th generation edge. A bound for the rate of convergence of $m_{j}$ has been obtained in [2]. Sharp estimates for certain classes of triangles have been given in [5]. In this paper we prove that $m_{j} \leqslant \sqrt{3} 2^{-j / 2} m_{0}$, if $j$ is even, and that $m_{j} \leqslant \sqrt{2} 2^{-j / 2} m_{0}$, if $j$ is odd, with equality for equilateral triangles. We prove, moreover, the following geometrically interesting fact: the (infinite) family of $U V W$ contains only finitely many similarity types.

Definition. If $\Delta$ is a triangle, then $\phi(\Delta)=($ area of $\Delta) / l^{2}(\Delta)$, where $l(\Delta)$ is the length of the longest edge of $\Delta . \mathscr{F}_{0}(\Delta)$ is the collection of even generation descendants of $\Delta$, and $\mathscr{F}_{1}(\Delta)$ is the collection of odd generation descendants.

Since our bisection process in particular bisects areas, in order to find out about $m_{j}$, it is enough to know how the dimensionless quantity $\phi(\Delta)$ behaves under bisection of triangles. Our results will be proved by an induction on $\phi$. It is necessary to deal first with acute angled triangles, then with obtuse triangles. The squares of side-lengths needed in this paper are all calculated by straightfoward use of the law of cosines.

2. Acute Triangles. Let $\Delta=U V W$ be an acute angled triangle, with $V W$ a longest edge. Write $\|U V\|^{2}=p,\|U W\|^{2}=q$. For convenience let $\|V W\|^{2}=1$, and assume $p \leqslant q \leqslant 1$. Bisect edge $V W$ at $A$. $U W$ is then the longest edge of $U A W$. Bisect it at $B$. Then $U A$ is the longest edge of $U B A$. There are now three different possibilities to consider.

Received June 3, 1980.

1980 Mathematics Subject Classification. Primary 51M20; Secondary 65N50, 65L50. 
Possibility 1. $U V$ is a longest edge of $U V A$. Examination of Figure 1 shows that bisection of $U A B$ and of $U V A$ gives rise to triangles similar to already occurring triangles, and so (up to similarity) $\mathscr{F}_{0}(\Delta)$ contains only $U V W$ and $U A B$, while $\mathscr{F}_{1}(\Delta)$ only contains $U V A$ and $U A W$.

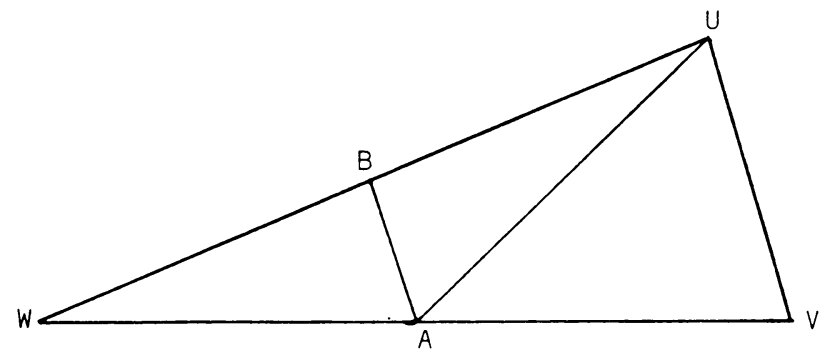

Figure 1

Since $\|U A\|^{2}=\frac{1}{4}(2 p+2 q-1), \phi(U A B)=\phi(\Delta) / 2 p+2 q-1$. But since $p+q$ $\geqslant 1$ (from the acuteness of $\Delta$ ), using elementary linear programming, we find that $\phi(U A B) \geqslant \frac{1}{3} \phi(\Delta)$, with equality if $\Delta$ is equilateral. It is easy to see that $\phi(U V A)$ and $\phi(U A W)$ are both $\geqslant \frac{1}{2} \phi(\Delta)$.

Since $\Delta$ is acute, $\|A V\| \leqslant\|A U\|$, so if Possibility 1 does not hold, $A U$ is a longest edge of $U V A$. Bisect $A U$ at $C$. It is not hard to show that $A V$ is a longest edge of $C V A$. Bisect $A V$ at $D$. We have reached the position illustrated in Figure 2.

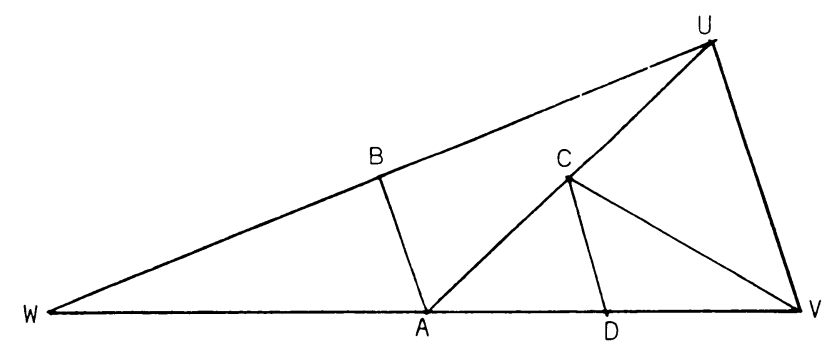

Figure 2

There are two possibilities now left.

Possibility 2. $U V$ is a longest edge of $U V C$. Examination of Figure 2 will show that further bisection produces triangles similar to already occurring triangles. So (up to similarity), $\mathscr{F}_{0}(\Delta)$ consists of $U V W, U A B, U V C$, and $C V A$, and $\mathscr{F}_{1}(\Delta)$ consists of $U V A, U A W$, and $C V D$. Since $U A$ is a longest edge of $U V A, \frac{1}{4}(2 p+2 q-1) \geqslant p$, so $q \geqslant p+\frac{1}{2}$. Elementary linear programming now gives $\phi(U A B) \geqslant \frac{1}{2} \phi(\Delta)$, and $\phi(U V C) \geqslant \frac{1}{2} \phi(\Delta)$. Of course $\phi(C V A)=\phi(\Delta)$. It turns out that $\|C V\|^{2}=$ $\frac{1}{16}(6 p-2 q+3)$. Linear programming now gives $\phi(C V D) \geqslant \frac{1}{2} \phi(\Delta)$. Similarly, we find that $\phi(U V A) \geqslant \phi(\Delta)$, and $\phi(U A W) \geqslant \frac{1}{2} \phi(\Delta)$.

If $U V$ is not the longest edge of $U V C$, there remains only

Possibility 3. $C V$ is a longest edge of $U V C$. So $\frac{1}{16}(6 p-2 q+3) \geqslant p$, that is $q \leqslant 3 / 2-5 p$. Then (up to similarity), $\mathscr{F}_{0}(\Delta)$ consists of $U V W, U A B$, and $\mathscr{F}_{1}(U V A)$, while $\mathscr{F}_{1}(\Delta)$ consists of $U A W$ and $\mathscr{F}_{0}(U V A)$. As usual, $\phi(U A W) \geqslant \frac{1}{2} \phi(\Delta)$. Since $q \leqslant 3 / 2-5 p$, linear programming gives $2 p+2 q-1 \leqslant \frac{6}{5}$. So $\phi(U A B) \geqslant \frac{5}{6} \phi(\Delta)$, 
while $\phi(U V A) \geqslant \frac{5}{3} \phi(\Delta)$. So $U V A$ is much "fatter" than $\Delta$. This enables us to push through an induction.

LEMMA 1. Let $\Delta$ be an acute triangle. Then the family of $\Delta$ contains only finitely many similarity types. If $\Gamma$ is in $\mathscr{F}_{0}(\Delta), \phi(\Gamma) \geqslant \frac{1}{3} \phi(\Delta)$. If $\Gamma$ is in $\mathscr{F}_{1}(\Delta), \phi(\Gamma) \geqslant \frac{1}{2} \phi(\Delta)$.

Proof. We show that if our assertions hold whenever $\phi(\Delta) \geqslant\left(\frac{3}{5}\right)^{n}$, they hold whenever $\phi(\Delta) \geqslant\left(\frac{3}{5}\right)^{n+1}$. So suppose $\Delta=U V W$ is acute and $\phi(\Delta) \geqslant\left(\frac{3}{5}\right)^{n+1}$. If $\Delta$ satisfies Possibility 1 or Possibility 2 , then, by our earlier calculations, $\Delta$ certainly satisfies our lemma. So suppose that $\Delta$ falls under Possibility 3. The elements of $\widetilde{F}_{1}(\Delta)$ are, up to similarity, $U A W$ (and $\left.\phi(U A W) \geqslant \frac{1}{2} \phi(\Delta)\right)$ together with $\mathscr{F}_{0}(U V A)$. But since $\phi(U V A) \geqslant \frac{5}{3} \phi(\Delta)$, by induction assumption $U V A$ satisfies our lemma, so if $\Gamma$ is in $\mathscr{F}_{0}(U V A), \phi(\Gamma) \geqslant \frac{1}{3} \phi(U V A) \geqslant \frac{5}{9} \phi(\Delta)>\frac{1}{2} \phi(\Delta)$. The same sort of calculation shows that under Possibility 3 , if $\Gamma$ is in $\mathscr{F}_{0}(\Delta), \phi(\Gamma) \geqslant \frac{1}{3} \phi(\Delta)$, indeed $\phi(\Gamma) \geqslant \frac{5}{6} \phi(\Delta)$. This completes the induction.

The inequalities for $\phi$ are sharp, for if $\Delta$ is equilateral, no improvement is possible. One cannot expect to make significant improvements on estimates for $\mathscr{F}_{1}(\Delta)$. But our proof shows that for the "general" acute triangle (Possibility 3 ), if $\Gamma$ is in $\mathscr{F}_{0}(\Delta)$, $\phi(\Gamma) \geqslant \frac{5}{6} \phi(\Delta)$.

3. Obtuse Triangles. Suppose now we are "bisecting" a triangle $\Delta=U V W$, where as usual $\|V W\|^{2}=1,\|U W\|^{2}=q,\|U V\|^{2}=p, p \leqslant q<1$, and where the angle $V U W$ is $\geqslant 90^{\circ}$. Bisect $V W$ at $A$. Then $U W$ is the longest edge of $\triangle U A W$. Bisect it at $B$ (see Figure 1).

LEMMA 2. If $\Delta$ is obtuse, the family of $\Delta$ contains only finitely many similarity types. If $\Gamma$ is in $\mathscr{F}_{1}(\Delta), \phi(\Gamma) \geqslant \frac{1}{2} \phi(\Delta)$. If $\Gamma$ is in $\mathscr{F}_{0}(\Delta), \phi(\Gamma) \geqslant \frac{1}{3} \phi(\Delta)$.

Proof. Let $\frac{1}{2} \leqslant \lambda<1$. We prove that if our result holds for all obtuse triangles $\Delta$ such that the smallest angle of $\Delta$ has cosine $\leqslant \sqrt{\lambda}$ and such that $\phi(\Delta) \geqslant \lambda^{n}$, then the result holds for all such $\Delta$ with $\phi(\Delta) \geqslant \lambda^{n+1}$. So suppose that $\phi(\Delta) \geqslant \lambda^{n+1}$, and that $\Delta$ has smallest angle $\alpha$, where $\cos ^{2} \alpha \leqslant \lambda$. If the angle $B A U(=A U V)$ is $\geqslant 90^{\circ}$, there is no problem. For it is easy to see that all angles of triangles $U A B, U V A$ are $\geqslant \alpha$. But

$$
\phi(U A B)=\frac{1}{q} \phi(\Delta) \geqslant \frac{1}{\cos ^{2} \alpha} \phi(\Delta) \geqslant \lambda^{n}
$$

Also $\phi(U V A)=2 \phi(\Delta) \geqslant 2 \lambda^{n+1} \geqslant \lambda^{n}$ since $\lambda \geqslant \frac{1}{2}$. Now $\mathscr{F}_{1}(\Delta)$ consists, up to similarity, of $U A W, \mathscr{F}_{1}(U A B)$, and $\mathscr{F}_{0}(U V A)$. By induction assumption, if $\Gamma$ is in $\mathscr{F}_{1}(U A B)$, then $\phi(\Gamma) \geqslant \phi(U A B) \geqslant \frac{1}{2} \phi(\Delta)$, while if $\Gamma$ is in $\mathscr{F}_{0}(U V A), \phi(\Gamma)$ $\geqslant \frac{1}{3} \phi(U V A)=\frac{2}{3} \phi(\Delta)>\frac{1}{2} \phi(\Delta)$. Elements of $\mathscr{F}_{0}(\Delta)$ are dealt with in the same way.

So it remains to see what happens if the angle $B A U$ is $\leqslant 90^{\circ}$. If $U V$ is the longest edge of $U V A$, the family of $\Delta$ has at most four similarity types, and a quick computation yields the result. Otherwise, $\phi(U V A)=2 \phi(\Delta)$, and of course $\phi(U A B)$ $\geqslant \phi(\Delta)$, and our result follows quickly from Lemma 1 .

The estimate for $\mathscr{F}_{1}(\Delta)$ cannot be significantly improved. But by a closer analysis of the possibilities that arise when the angle $U A B$ is acute, one can show that in fact if $\Gamma$ is in $\mathscr{F}_{0}(\Delta), \phi(\Gamma) \geqslant \phi(\Delta)$. 
4. Summary, Problems. By combining Lemma 1, Lemma 2, and the fact that area goes down by a factor of 2 each generation we obtain:

THEOREM. Under the bisection process, the family of a triangle falls into finitely many similarity classes. If $j$ is even, $m_{j} \leqslant \sqrt{3} 2^{-j / 2} m_{0}$. If $j$ is odd, $m_{j} \leqslant \sqrt{2} 2^{-j / 2} m_{0}$.

Both estimates are sharp, for we have equality when the triangle is equilateral. If the starting triangle is far from being equilateral, the bounds for $m_{j}$ when $j$ is even can be improved. By examining the details of the proof, one can find an upper bound for the number of similarity types in the family of $\Delta$, say as a function of $\phi(\Delta)$. But there appears to be nothing very interesting left to do for triangles.

But one can raise similar problems in a much more general setting. Let $A_{1}, A_{2}, \ldots, A_{n}$ be a configuration of $n+1$ points in $d$-dimensional space. Suppose $\left\|A_{0}-A_{1}\right\| \geqslant\left\|A_{i}-A_{j}\right\|$ for all $i, j$. Then the configuration gives birth to two daughter configurations $A_{0},\left(A_{0}+A_{1}\right) / 2, A_{2}, \ldots, A_{n}$ and $\left(A_{0}+A_{1}\right) / 2$, $A_{1}, A_{2}, \ldots, A_{n}$. One can define $m_{j}$ as for triangles and ask about the behavior of $m_{j}$. It seems reasonable to conjecture that $m_{j}=O\left(2^{-j / n}\right)$. One can make the even stronger conjecture that up to similarity any configuration has a finite family.

Already for four points in general position in 3-dimensional space, the problems seem difficult. We have a proof of the "finite family" conjecture for certain classes of tetrahedra. For example, it turns out that if a tetrahedron is nearly equilateral and the second largest edge is opposite the longest edge, then the family of the tetrahedron falls into $\leqslant 37$ similarity classes. (The condition "nearly equilateral" is a little complicated to describe briefly, but, for example, it is satisfied if all edge lengths are within $5 \%$ of each other.)

Department of Mathematics

University of British Columbia

Vancouver, B.C., Canada V6T IW5

1. C. Harvey \& F. Stenger, "A two dimensional analogue to the method of bisections for solving nonlinear equations," Quart. Appl. Math., v. 33, 1976, pp. 351-368.

2. R. B. KeARFotT, "A proof of convergence and an error bound for the method of bisection in $R^{n}$," Math. Comp., v. 32, 1978, pp. 1147-1153.

3. K. SiKorsKi, "A three-dimensional analogue to the method of bisections for solving nonlinear equations," Math. Comp., v. 33, 1979, pp. 722-738.

4. F. Stenger, "Computing the topological degree of a mapping in $n$-space," Numer. Math., v. 25 , 1975, pp. 23-38.

5. M. STYNES, "On faster convergence of the bisection method for certain triangles," Math. Comp., v. 33. 1979, pp. 717-721. 